\title{
SPACE CHARGE AND BEAM STABILITY OF THE PROPOSED FERMILAB PROTON DRIVER IN PHASE I
}

\author{
K.Y. Ng, FNAL, Batavia, IL 60510, USA and B. Zotter, CERN, CH-1211, Geneva 23, Switzerland
}

\section{Abstract}

Issues concerning beam stability of the proposed Fermilab proton driver are studied in its Phase I. Although the betatron tune shifts are dominated by space charge, these shifts are less than 0.25 and will therefore not drive the symmetric and antisymmetric modes of the beam envelope into instability. The longitudinal space-charge force is large and inductive inserts may be needed to compensate for the distortion of the rf potential. Although the longitudinal impedance is space-charge dominated, it will not drive any microwave instability, unless the real part of the impedance coming from the inductive inserts and wall resistivity of the beam tube are large enough. The design of the beam tube is therefore very important in order to limit the flow of eddy current and keep wall resistivity low. The transverse impedance is also space-charge dominated. With the proton driver operated at an imaginary transition gamma, however, Landau damping will never be canceled and beam stability can be maintained with negative chromaticities.

\section{INTRODUCTION}

The proposed Fermilab proton driver will be a $15 \mathrm{~Hz}$ rapid-cycling high-intensity synchrotron that serves a number of purposes in the Fermilab high energy physics program. In Phase I Stage 1, the proton driver, of circumference $C=2 \pi R=711.3 \mathrm{~m}$, accelerates $N=3 \times 10^{13}$ protons in $n_{b}=126$ bunches with a $53 \mathrm{MHz}$ rf system from the injection kinetic energy (K.E.) of $400 \mathrm{keV}$ to the K.E. of $12 \mathrm{GeV}$ to be injected into the Main Injector. In Stage 2, the $3 \times 10^{13}$ protons are divided into $n_{b}=18$ bunches and accelerated with a $75 \mathrm{MHz}$ rf system to the K.E. of $16 \mathrm{GeV}$ for the production of muons destined for a neutrino factory. Because of its low-energy injection, space-charge effects are significant. Because of its high intensity, collective single and multi-bunch instabilities are important issues. In Stage 2 , there is a bunch compression by rotation before extraction, which will be covered in detail in Ref. [1].

\section{SPACE CHARGE AND TUNE SHIFTS}

The stainless steel vacuum chamber is of elliptical cross section with horizontal/vertical radii $w=119 \mathrm{~mm}$ and $h=63 \mathrm{~mm}$. The magnet pole faces have vertical half gap $g=64 \mathrm{~mm}$ covering about $\kappa=50 \%$ of the ring. The bare horizontal/vertical tunes are $\nu_{0 x, y}=12.428 / 11.380$ so that the average betatron functions are $\left\langle\beta_{x, y}\right\rangle=9.109 / 9.948 \mathrm{~m}$. The average dispersion is $\langle D\rangle=1.247 \mathrm{~m}$. The beam has normalized $90 \%$ emittance $\epsilon_{x, y}=60 \times 10^{-6} \pi \mathrm{m}$. Thus, at injection the beam has elliptical cross section with horizontal/vertical radii $a_{x, y}=23.2 / 24.2 \mathrm{~mm}$, where the full energy spread is $7 \times 10^{-4}$ throughout the cycle. The rf

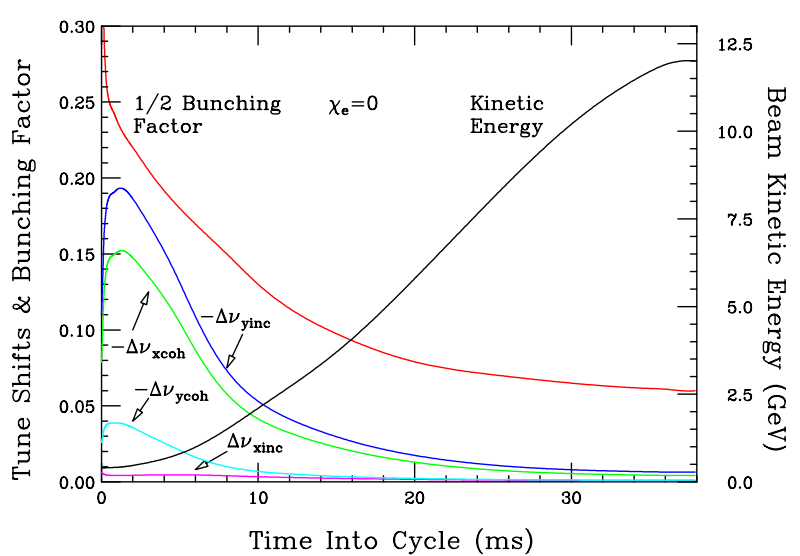

Figure 1: (color) Bunching factor, energy and tune shifts during one acceleration cycle.

frequency is $53 \mathrm{MHz}$. An acceleration cycle has been designed [2] with K.E. and bunching factor $B_{f}$ as shown in Fig. 1. With $z=x, y$, the incoherent and coherent tune shifts can be computed according to [3]

$$
\begin{aligned}
& \Delta \nu_{z \text { inc }}=-\frac{N r_{p} R}{\pi \beta^{2} \gamma \nu_{0 z}} {\left[\frac{\gamma^{-2}-\chi_{e}}{B_{f} a_{z}\left(a_{x}+a_{y}\right)}+\right.} \\
&\left.+\left(\beta^{2}+\frac{\gamma^{-2}-\chi_{e}}{B_{f}}\right) \frac{\epsilon_{1 z}}{h^{2}}+\kappa \beta^{2} \frac{\epsilon_{2 z}}{g^{2}}\right], \\
& \Delta \nu_{z \mathrm{coh}}=-\frac{N r_{p} R}{\pi \beta^{2} \gamma \nu_{0 z}}\left[\frac{\gamma^{-2}-\chi_{e}}{B_{f}} \frac{\xi_{1 z}}{h^{2}}+\beta^{2} \frac{\epsilon_{1 z}}{h^{2}}+\kappa \beta^{2} \frac{\epsilon_{2 z}}{g^{2}}\right],
\end{aligned}
$$

where $\gamma$ and $\beta$ are the relativistic factors of the protons, $r_{p}$ the proton classical radius, and $\chi_{e}$ the neutralization factor. In above, $\epsilon_{1 z, 2 z}$ are the Laslett incoherent electric/magnetic image coefficients, while $\xi_{1 z}$ are the coherent electric image coefficients. For the elliptical beam pipe, we have $\epsilon_{1 y}=$ $-\epsilon_{1 x}=0.1669, \xi_{1 x}=0.0948, \xi_{1 y}=0.5956$. For the magnetic coefficients, parallel-plate approximation gives $\epsilon_{2 y}=$ $-\epsilon_{2 x}=\pi^{2} / 24$. The tune shifts are plotted in Fig. 1. Stage 2 will be similar in the early part of the cycle. We see that the tune shifts reach their maxima about 1-2 ms after injection and capture in the rf field, when the bunching factor decreases sharply from $B_{f}=0.9$ but the beam energy is still low. We also see that the incoherent tune shifts are less than 0.25 , although space charge is dominating. However, if the transverse distribution is not uniform but peaked, the spacecharge tune shift can be higher. Simulation shows that the incoherent vertical tune shift is slightly above 0.25 and the horizontal slightly below [4]. But this is of no concern, because it is the coherent tune shifts that play a role of resonant instability. Dipole errors cannot deflect the beam coherently [5]. Quadrupole errors will shift the 2 quadrupole modes by $\frac{3}{4}$ and $\frac{1}{2}$ of the incoherent space-charge tune shift which is less than 0.2 . Thus, the choice of the current bare tunes will not lead to a half integer resonance. 


\section{SINGLE BUNCH INSTABILITIES}

\subsection{Broad-band impedance estimates}

The largest impedances in the proton driver at low energies are due to space charge [6]:

$$
\begin{gathered}
\frac{Z_{\|}^{s c}}{n}=-j \frac{Z_{0}}{2 \beta \gamma^{2}} g_{f}=-384 j \Omega, \quad Z_{\perp x, y}^{s c}= \\
-j \frac{2 R Z_{0}}{\beta^{2} \gamma^{2}}\left[\frac{1}{a_{z}\left(a_{x}+a_{y}\right)}-\frac{\xi_{1 z}-\epsilon_{1 z}}{b^{2}}\right]=-70 j / 63 j \mathrm{M} \Omega / \mathrm{m},
\end{gathered}
$$

where $Z_{0} \approx 377 \Omega$. With the vacuum chamber made of Inconel having resistivity $\rho_{c}=129 \mu \Omega \mathrm{cm}$ and relativity permeability $\mu_{r}=1.002$, the wall resistivity is, at injection,

$$
\frac{Z_{\|}^{r w}}{n}=(1+j) \beta \mu_{r} Z_{0} \frac{\delta_{h}}{2 h}=1.48(1+j) n^{-1 / 2} \Omega .
$$

The transverse impedance is found by multiplication with $2 R /\left(\beta h^{2}\right)$ or $0.033 / 0.118(1+j)\left|n-\nu_{x, y}\right|^{-1 / 2} \mathrm{M} \Omega / \mathrm{m}$. In above, $\delta_{h}=\sqrt{2 \rho_{c} /\left(\omega \mu_{0} \mu_{r}\right)}$ is the skin depth.

Other sources of broad-band impedance are the rf cavities loaded with ferrite (or Finemet), kicker tanks, bellows, and variations of vacuum cross section. However, they will contribute mainly inductive impedance at low frequencies.

\subsection{Eddy Current and shielding of em fields}

To prevent excessive eddy current losses, the metallic vacuum chamber can be divided into narrow strips, similar to the wire-cage used in ISIS [7]. The eddy current power loss per unit length in a metal strip of width $w$, thickness $t$ and conductivity $\sigma_{c}$, at right angle to a changing magnetic field with time derivative $\dot{B}$, is approximately given by $\sigma_{c} t w^{3} \dot{B}^{2} / 12$. Since it is proportional to the third power of the width, it can be reduced from, for example, over $8 \mathrm{~kW} / \mathrm{m}$ for a $1.3 \mathrm{~mm}$ thick, $22 \mathrm{~cm}$ wide, elliptic Inconel chamber to a few $\mathrm{W} / \mathrm{m}$ by replacing it with $2 \times 50$ copper strips of $4 \mathrm{~mm}$ width and 50-100 $\mu \mathrm{m}$ thickness.

When the vacuum chamber is thin enough, we have to worry about the field of the beam penetrating into the magnetic laminations, where the large relative permeability $\mu_{r}$ translates into large $\operatorname{Re} Z_{\|}^{r w}$. For this purpose, a screen or liner has been proposed, similar to the radiation shield for the LHC [8]. To minimize eddy current losses, the screen should be made as thin as possible. Assuming rotational symmetry, the shielding condition in the longitudinal direction of a screen of thickness $t$ at radius $b$, with skin depth $\delta_{b} \gg t$, and an outer wall at radius $d$, with skin depth $\delta_{d}$, can be written as [9]

$$
\frac{t}{\delta_{b}} \gg \frac{\beta^{2} \gamma^{2}}{2 \ln (d / b)} \frac{\delta_{b}}{b} .
$$

In the transverse direction, the shielding condition under the same assumption is the same but without the factor of $2 \ln (d / b)$. Hence, once longitudinal shielding is achieved, transverse shielding is guaranteed.

For higher energies, the shielding criterion becomes $t / \delta_{b} \gg(d / b)\left(\delta_{b} / \delta_{d}\right)$, which is difficult to fulfill. However, at higher energies the beam is more stable and the space charge part of the impedance is strongly reduced.

\subsection{Longitudinal stability criteria}

The Boussard-Keil-Schnell criterion for longitudinal bunched beam stability is [10]

$$
\frac{\left|Z_{\|}\right|}{n}<F \frac{|\eta| B_{f} E}{e \beta^{2} I_{b}}\left[\frac{\Delta E}{E}\right]_{\mathrm{FWHM}}^{2},
$$

where $I_{b}$ is the average bunch current, $\eta=\gamma_{t}^{-2}-\gamma^{-2}$ the slip factor, and the form factor $F \approx 1$ for a parabolic distribution distribution, but is actually very much larger for a space-charge dominated beam. With a transition gamma of $\gamma_{t}=27.71 j$ and $F=1$, the stability limit starts from $24.95 \mathrm{k} \Omega$ at injection and drops monitonically to $0.16 \mathrm{k} \Omega$ at extraction, and is at all times larger than the space-charge impedance $\left|Z_{\|}^{s c} / n\right|$. For Phase I Stage 2, the same current is divided into only 18 bunches so that the threshold is 7 times smaller, but is still much larger than $\left|Z_{\|}^{s c} / n\right|$ at all times.

\subsection{Transverse stability criteria}

The Boussard-Keil-Schnell-like criterion for transverse bunched beam stability is (for $z=x, y$ ) [11],

$$
\left|Z_{\perp z}\right|<F \frac{4 B_{f} E}{e \beta\left\langle\beta_{z}\right\rangle I_{b}}\left[\frac{\Delta E}{E}\right]_{\mathrm{FWHM}}\left|S_{z}\right|,
$$

where the effective chromaticity is $S_{z}=\left(n-\nu_{z}\right) \eta+\xi_{z}$, with $n$ an arbitrary integer and $\xi_{z}$ the chromaticity. Instability occurs only for slow waves with $n>\nu_{z}$. The form factor $F$ depends on the transverse particle distribution, but is large compared to unity for a space-charge dominated impedance as in the longitudinal case. Here, $\eta$ is always negative. If also the chromaticity $\xi_{z}$ is negative, the two terms add and cannot cancel. The lowest value of the spread is obtained for $n$ just above the tune $\nu_{z}$. Since also $|\eta| \approx \frac{1}{2}$, the first term in $S_{z}$ can be neglected if $\left|\xi_{z}\right|$ is large enough. For $F=\left|S_{z}\right|=1$, the transverse impedance threshold dips down to $11 \mathrm{M} \Omega / \mathrm{m}$ in Stage 1 about $5 \mathrm{~ms}$ into the acceleration cycle. This is very much larger than $\left|Z_{\perp z}^{r w}\right|$ even when $|n-\nu|=1$, and requires $\xi_{z} \approx-4$ to cope with the space-charge impedance. In Stage 2 , however, the threshold is 7 times smaller and is still much larger than $\left|Z_{\perp z}^{r w}\right|$, but requires $F\left|S_{z}\right| \approx 28$ to overcome the space-charge impedance. To safeguard stability, one may blow up the longitudinal emittance so as to attain a larger energy spread, thus enhacing Landau damping.

\subsection{Inductive Inserts}

To compensate for the large space-charge distortion of the rf wave form, inductive inserts can be installed for compensation. However, the ferrite cores in the inserts can have large $\mathcal{R} e Z_{\|}$and may drive the beam into microwave instability. Thus, we should choose ferrite with small losses or we may lower the ferrite losses by heating the inserts [12].

\section{COUPLED-BUNCH INSTABILITIES}

\subsection{Longitudinal stability criterion}

Longitudinal coupled-bunch instability are mostly driven by the parasitic resonances in the rf system. The $53 \mathrm{MHz}$ rf cavities used in the present Fermilab booster and the past 
Fermilab Main Ring have a large number of higher-order resonances and have driven coupled-bunch instabilities in these two machines. However, careful passive detuning of these resonances has gotten rid of most instabilities. Since the total number of protons in the proton driver will be about 3 times that in the present booster, the coupled-bunch growth rates will be 3 times as fast. As a result, past experience in passive damping of parasitic resonances may not be sufficient to cope with the coupled-bunch instabilities. Further lowering of growth rates can be achieved by blowing up the longitudinal emittance of the bunches which alters the form factor by decreases the coherency inside the bunches, or by installing a fast bunch-by-bunch damper.

In Stage 2, $75 \mathrm{MHz}$ Finemet rf cavities with low quality factor $Q \approx 10$ will be used. As a result, there will not be any parasitic resonances inside the cavities to drive coupledbunch instabilities, either longitudinally or transversely.

\subsection{Transverse stability criterion}

Possible transverse coupled-bunch instabilities driven by parasitic resonances in the $53 \mathrm{MHz}$ rf cavities can be cured by detuning passively as in the longitudinal situation, or by blowing up the longitudinal emittance of the bunches. However, transverse coupled-bunch instabilities can also be driven by the resistive wall impedance at the frequency $\left(\left[\nu_{z}\right]-1\right) f_{0}$, where $f_{0}$ is the revolution frequency and $\left[\nu_{z}\right]$ the noninteger part of the betatron tune. For $n_{b}$ bunches each with average current $I_{b}$, the growth rate is [11]

$$
\frac{1}{\tau}=-\frac{1}{m+1} \frac{e n_{b} I_{b} c}{4 \pi \nu_{z} E} \sum_{k} \operatorname{Re} Z_{\perp z}\left(\omega_{k}\right) F_{m}^{\prime}\left(\omega_{k}-\chi_{z}\right)
$$

where $\chi_{z}=-\xi_{z} \omega_{0} \tau_{L} / \eta$ is the chromatic phase shift across the full length $\tau_{L}$ of the bunch. The frequency spectrum for the $m$-th mode of transverse oscillations with coupled bunch mode number $n\left(0 \leq n<n_{b}\right)$ is given by $\omega_{k}=k n_{b}$ $+n+\nu_{z}+m \nu_{s}$. The form factor $F^{\prime}(\omega)$ expresses the cancellation occurring in the summation over both positive and negative frequencies of the impedance, weighted by the spectrum of the $m$-th mode of oscillation. In this situation, however, the contribution is dominated by only one spectral line at the above low frequency and $F^{\prime} \approx 0.8$. Fortunately the ring is small; with the resistive wall impedance computed in Sec. 3.1, the growth times are just 137/37 ms horizontally/vertically with $\xi_{z}=0$ at injection in both Stages 1 and 2 . The growth rate for mode $m=0$ can be lowered by operating at a negative chromaticity. However, this may introduce instabilities of mode $m \geq 1$ which can be damped by a tune spread of order $1 /\left(\omega_{0} \tau_{L}\right)$ that usually occurs naturally; thus a low-frequency damper may not be necessary.

\section{ELECTRON-PROTON INSTABILITY}

When a proton beam is partially neutralized, with fractional neutralization $\chi_{e}$, the electrons in it will start to oscillate transversely with the bounce frequency given by, for small amplitudes, $\omega_{e}^{2}=4 c^{2} r_{e} n_{p}$, where $r_{e}$ is the classical electron radius, and $n_{p}=N_{b} /\left(\pi a_{x} a_{y} L_{b}\right)$ is the volume density of the protons in a bunch with $N_{b}$ protons, cross sectional area $\pi a_{x} a_{y}$ and full length $L_{b}$. The oscillating electrons will excite the protons to oscillate with frequency $\omega_{p}^{2}=4 c^{2} r_{p} n_{e}=4 c^{2} r_{p} \chi_{e} n_{p}$. The coupled oscillations lead to a dispersion relation [13] for the e-p oscillation frequency $\omega$ as function of the azimuthal mode number $n$ :

$$
\left(\omega_{e}^{2}-\omega^{2}\right)\left[\left(\nu_{z} \omega_{0}\right)^{2}+\omega_{p}^{2}-\left(n \omega_{0}-\omega\right)^{2}\right]=\omega_{e}^{2} \omega_{p}^{2} .
$$

While the amplitudes of the heavier protons are small, those of the lighter electrons are larger and will grow rapidly, reach the vacuum chamber wall, and generate more electrons by secondary emission, which can lead to an avalanche effect called multipactoring. This may then lead also to emittance growth and/or beam loss of the protons.

e-p oscillations had been seen in the CERN ISR and had to be eliminated by better clearing and pumping. They are also seen in a number of proton storage rings, like the PSR at Los Alamos, where the beam current remained below the design goal for many years after many attempts to eliminate or at least reduce the number of electrons failed.

The most common method to reduce neutralization of a beam is to leave a gap in the train of bunches, which has also been foreseen for the Fermilab proton driver. A more active method is to install clearing electrodes, and eliminate the electrons by applying transverse electric fields. If the electrons are generated by vacuum, better pumping may helpbut not if the electrons are coming from the $H^{-}$stripping foil. Multipactor at the wall can be reduced by coating with a material with low secondary emission coefficient, such as Ti-N. If nothing else helps, a feedback system can be the solution. For the case of the LANL PSR, all these methods were tried and failed, and only a combination of higher $\mathrm{rf}$ voltage, sextupoles, partial wall coating and finally an inductive insert permitted to reach the design current.

For the present proton driver, the injection from the linac is in chopped beams. As the rf voltage is raised, the bunching factor decreases rapidly from $B_{f}=0.9$ to 0.44 in $2 \mathrm{~ms}$ and 0.18 one half into the acceleration cycle. Thus, there will be large bunch gaps so that trapped electrons should be cleared and hence no e-p instability is expected.

\section{REFERENCES}

[1] K.Y. Ng, these proceedings.

[2] Z. Qian, private communication.

[3] J. Laslett, BNL Report 7534, (1963) p. 234; J. Laslett and L. Resegotti, Proc. 6th Int. Conf. High Energy Acc., Cambridge 1967, P.150.

[4] C. Prior, private communication.

[5] R. Baartman, AIP Conf. Proc. 448 (1998) p. 56.

[6] D. Moehl, A. Sessler, Proc. 8th Int. Conf. High Energy Acc., CERN, 1971, p.334.

[7] I. Gardner et al., Part. Accel. 31, 227 (1990).

[8] F. Caspers et al., Proc. PAC New York, 1999, p. 1408.

[9] R. Gluckstern, CERN Report CERN 2000-011, 2000.

[10] E. Keil and W. Schnell, CERN/ISR-TH/69-48, 1969; D. Boussard. CERN Lab II/RF/75-2, 1975.

[11] F. Sacherer, CERN Report 77-13, 1977.

[12] K.Y. Ng, et al., these proceedings.

[13] B. Zotter, CERN-ISR-TH/71-58, 1971. 